\title{
NL1 expression level in Nrx1 $\beta$ and the excitability of PV interneurons in mice with POCD
}

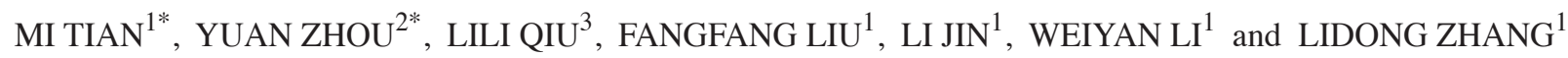 \\ Departments of ${ }^{1}$ Anesthesiology and ${ }^{2}$ Neurosurgery, Nanjing General Hospital; \\ ${ }^{3}$ Department of Anesthesiology, Zhongda Hospital Southeast University, Nanjing, Jiangsu 210000, P.R. China
}

Received May 29, 2018; Accepted January 10, 2019

DOI: 10.3892/etm.2019.7322

\begin{abstract}
Effects of NL1 on the expression level of neurexin-1 $\beta$ (Nrx1 $\beta$ ) and the excitability of parvalbumin (PV) interneurons in mice with postoperative cognitive dysfunction (POCD) were explored. Eighty 10-month-old C57BL/6 male mice were randomly divided into four groups: control group, control+empty vector group (Control+EV), anesthesia surgery+empty vector group (POCD+EV group) and anesthesia+NL1 overexpression group (POCD+NL1 group), ( $n=20$ per group). The behavioral differences of mice in the four groups were analyzed by means of open field test and fear conditioning test. The expression levels of NL1, PV and Nrx1 $\beta$ were detected by western blot analysis, and the binding between NL1 and Nrx1 $\beta$ was determined by co-immunoprecipitation. The expression level of postsynaptic density protein 95 (PSD95) in hippocampus and changes in the excitability of PV interneurons were further detected. Control and Control+EV groups had no significant difference in each index $(\mathrm{P}>0.05)$. Compared with Control+EV group, the percentage of cued freezing time in POCD+EV group decreased significantly, while percentage of cued freezing time was significantly increased in POCD+NL1 group and $\mathrm{POCD}+\mathrm{EV}$ group $(\mathrm{P}<0.01)$. The differences in freezing time were not statistically significant among the 4 groups in the tone-related fear test $(\mathrm{P}>0.05)$. Then NL1 was overexpressed in mice with POCD, the protein levels of PV, Nrx1 $\beta$ and PSD95 were subsequently increased, and the interaction between NL1 and $\operatorname{Nrx} 1 \beta$ protein was enhanced, dramatically increasing the excitability of PV interneurons. The overexpression of NL1
\end{abstract}

Correspondence to: Dr Weiyan Li or Dr Lidong Zhang, Department of Anesthesiology, Nanjing General Hospital, 305 Zhongshan East Road, Nanjing, Jiangsu 210000, P.R. China

E-mail: candytianmixixi@163.com

E-mail: ldzhang1968@163.com

*Contributed equally

Key words: NL1, postoperative cognitive dysfunction, Nrx1 $\beta$, parvalbumin, neuronal excitability can upregulate the expression levels of PV, Nrx1 $\beta$ and PSD95 in mice with POCD, enhance the interaction between NL1 and Nrx $1 \beta$ and further increase the excitability of PV interneurons, thus restoring the hippocampus-dependent memorial and cognitive impairment in POCD.

\section{Introduction}

The postoperative cognitive dysfunction (POCD) is a common central nervous system complication after anesthesia (1), which affects many aspects of cognition, such as memory, attention, information processing and cognitive flexibility $(2,3)$. POCD tends to occur in elderly patients. Studies have shown that the probability that patients aged over 65 are subjected to surgery is $>50 \%$. Due to the growing aging problem, the occurrence of POCD has also continuously increase with the rising number of elderly patients receiving operations (4). The occurrence of POCD will prolong the hospital stay of patients, affect the long-term life quality and even increase the mortality rate, which adds a heavy burden to the family and society. There are studies suggesting that POCD is related to the surgery, anesthesia and patients, and more likely to be affected synthetically by these three factors (5), but its specific mechanism remains unclear. Therefore, studying deeply the occurrence mechanism of POCD will provide new theoretical basis and potential intervention targets for its prevention and treatment, which is of great practical significance.

All the information processing in the brain involves synapses, the nerve impulses will not be transmitted among neurons without effective synapses, and the cognition and other nervous processes will also not be achieved. For the normal neural circuit function, the formation and differentiation of synapses appear to be particularly important (6). There is a complex connection between the formation and diverse differentiation of synapses, and both of them are dependent on the activity of synapse cell-adhesion molecules (CAMs). The activity of inhibitory interneurons is under the control of excitatory synapses and inhibitory synapses formed by glutamatergic neurons and GABAergic neurons $(7,8)$. When the excitatory signals of pyramidal cells decrease, the dysfunction of parvalbumin (PV) interneurons can be induced, weakening the inhibition of pyramidal cells, resulting in the imbalance of excitatory synapse/inhibitory synapse transmission and destroying the effective activities of neural network (9). 
CAMs include neurexins (NRXs), neuroligins (NLs), $\mathrm{N}$-cadherin, synaptic cell adhesion molecule-1 (SynCAM-1), ephrinB, and ephrinB receptor system (10). NRXs and NLs are important among them, their specific synapse functions have been researched most deeply up to now (11). The transsynaptic interaction of postsynaptic NLs with the presynaptic NRXs holds an important status in the synaptogenesis and synaptic integrity. NL1 is the CAM specifically located in the excitatory postsynaptic membrane and plays a significant role in the formation and function of excitatory synapses through the transsynaptic combination of presynaptic neurexin-1 $\beta(\operatorname{Nrx} 1 \beta)$, which can cause the differentiation of synapses and regulate the transmission of neurotransmitter among neurons (12). The decreased interaction between the two affects the function of excitatory synapses and the transmission of glutamatergic neurotransmitter and destroys the effective activities of neural network.

PV interneurons are the inhibitory GABAergic neurons, accounting for $40-50 \%$ in the total GABAergic neurons. They can release the GABAergic neurotransmitter, transmit the inhibitory signals to the pyramidal cells and control the excitability of pyramidal cells (13). Moreover, the pyramidal cells can also release the glutamatergic neurotransmitter, transmit the excitatory signals to the PV interneurons and activate the $\mathrm{PV}$ interneurons to release GABA. Such a process forms a neural circuit that regulates the effective activities of neural network (14).

Our previous study suggested that the downregulation of hippocampus NL1 can mediate the recruitment of postsynaptic glutamate receptors, regulate the release of synapse glutamate to affect the transmission of excitatory synapses and destroy the excitatory signals emitted by pyramidal cells to PV interneurons (unpublished data). This resulted in PV interneuron function impairment, weakening the inhibition of pyramidal cells, triggering the imbalance of excitatory synapse/inhibitory synapse transmission and finally leading to the occurrence of POCD.

\section{Materials and methods}

Laboratory animals. A total of 80, 10 month-old C57BL/6 healthy male mice of clean grade aged 10 weeks were purchased from the Laboratory Animal Center of Fudan University, and fed in the specific pathogen-free environment at room temperature of $25 \pm 2^{\circ} \mathrm{C}$ with free access to food and water. The study was approved by the Ethics Committee of Nanjing General Hospital (Nanjing, China).

Laboratory animal grouping. Mice were randomly divided into four groups: control group, control+empty vector group (control+EV), anesthesia surgery+empty vector group (POCD+EV group) and anesthesia+NL1 overexpression group (POCD+NL1 group), 20 in each group.

Establishment of the animal model. Animals were reared for 14 days to adapt to the environment. Animals were then randomly divided into 4 groups: normal control group $(n=20$, no anesthesia and surgery) and Control+EV group ( $\mathrm{n}=20$ mice, in vivo transfection of empty vector), POCD+EV group $(n=20$, anesthesia, surgery and empty vector transfection) and POCD+NL1 group $(n=20$, anesthesia, surgery and NL1 expression vector transfection). POCD modeling: anesthesia chamber was pre-charged with $1.5 \%$ isoflurane $+100 \%$ oxygen ( $21 / \mathrm{min}$ oxygen flow) for $15 \mathrm{~min}$, and then mice were put into the box to maintain anesthesia for $30 \mathrm{~min}$. After the mouse right reflex disappeared, the right lateral position was taken to avoid aspiration and maintain airway patency. Gas concentration in the anesthesia chamber was continuously monitored by a continuous monitoring multi-parameter anesthetic gas monitor, and the vital signs were checked to maintain the normal anesthetic depth. During the operation, a $1 \mathrm{~cm}$ long incision was made along the midline of the abdomen to perform an abdominal exploration and ensure that the direction of the bowel did not change. After exploration, the muscle fascia and skin were sutured using 5-0 and 4-0 sterile surgical sutures. The sterile conditions were ensured throughout the operation. After operation, the mice were returned to the warm table and kept warm. Mice in normal control group were not treated except the injection of solvent in an equal volume.

In vivo transfection. A total of $60 \mathrm{POCD}$ mice were divided randomly and equally into the POCD+EV and POCD+NL1 groups. Nucleic acids $(12.5 \mu \mathrm{g})$ were diluted into $1 \mu \mathrm{g} / \mu \mathrm{l}$ with an appropriate amount of endotoxin-free pure water, and added with $12.5 \mu \mathrm{l}$ water and $25 \mu \mathrm{l} 10 \%$ glucose solution (w/v), and the final volume of $50 \mu \mathrm{l}$ was mixed evenly. Twenty-five microliters of Entranster ${ }^{\mathrm{TM}}$-in vivo reagent was diluted with $25 \mu \mathrm{l}$ of $10 \%$ glucose solution to a final volume of $50 \mu \mathrm{l}$. After that, the diluted transfection reagent was immediately added into the diluted nucleic acid solution and mixed evenly, obtaining the transfection complex. After being placed at room temperature for $15 \mathrm{~min}$, the transfection complex was injected into mice in POCD+NL1 group via the caudal vein. Mice in POCD+EV group were injected with an equal amount of normal saline.

Open field test. The open field test is a method used to evaluate the autonomous behaviors, exploratory behaviors and tension degree of experimental animals in the new environment, in which the frequency and duration of certain behaviors of experimental animals in the new environment are used to reflect their autonomous behaviors and exploratory behaviors in the unfamiliar environment, and the times of urination is used to present the tension degree. The open field test was performed in a quiet environment: Mice were placed in the center of the box bottom, accompanied by shouting and timing using the image automatic monitoring system (Jiangsu SANS Biological Technology Co., Ltd., Jiangsu, China). After observation for a certain period of time based on experimental requirements (generally 3-5 $\mathrm{min}$ ), shouting was terminated. The inner wall and bottom of the square box were cleaned to prevent the residual information of animals (such as the urine, feces and odor) in the last test from affecting the results of the next test. The test was performed again after mice were replaced.

Fear conditioning test. At day 1 of the test, mice were put into an experimental box with electric metal fences on the bottom. After mice adapted to the environment for $3 \mathrm{~min}$, they were stimulated by the single-frequency sound $(3.0 \mathrm{kHz}$, $65 \mathrm{Db}, 30 \mathrm{sec}$ ), as well as the inevitable electric foot-shock $(0.7 \mathrm{~mA}, 2 \mathrm{sec})$ in the last $2 \mathrm{sec}$ at the same time. Sound and 
electric shock were terminated simultaneously. After the test, mice were kept in the box for $3 \mathrm{~min}$, and then continued to be fed in the cage. The box bottom was wiped with $75 \%$ alcohol after each test. After $24 \mathrm{~h}$, mice with fear conditioning already established were placed into the original box without any stimulation. The contextual freezing mainly used to reflect the hippocampus-dependent memory was recorded within $3 \mathrm{~min}$. After $2 \mathrm{~h}$, the environment in the box was changed (a white board was put on the bottom of box to change the color of the box wall), and mice were placed into it again to adapt to the environment, after which they were stimulated by the same intensity of sound for $3 \mathrm{~min}$. The cued freezing mainly used to reflect the non-hippocampus-dependent memory was recorded within $3 \mathrm{~min}$. The freezing was defined as no other motor behaviors except breathing. The percentages of time of contextual freezing and cued freezing were recorded.

Western blot analysis. Detection of NL1, PV and Nrx1 $\beta$ protein expression levels in hippocampal tissues: At $3 \mathrm{~h}$ after fear conditioning test, mice were decapitated rapidly, and hippocampal tissues were taken and added with tissue lysis solution, followed by grinding and homogenization on ice. After centrifugation at $3,000 \mathrm{xg}$ at $4^{\circ} \mathrm{C}$ for $8 \mathrm{~min}$, the supernatant was taken, and the protein concentration was detected using the bicinchoninic acid (BCA) method. After the protein was separated via $12 \%$ polyacrylamide gel electrophoresis, it was transferred onto a polyvinylidene fluoride (PVDF) membrane using the semi-dry method, and sealed with $5 \%$ skim milk powder at room temperature for $1 \mathrm{~h}$. Then the band was incubated with the primary antibodies of NL1 (diluted at 1:2,000; cat. no. ab186279), PV (diluted at 1:1,000; cat. no. ab27853), Nrx1 $\beta$ (diluted at 1:2,000; cat. no. ab2869) and $\beta$-actin (diluted at 1:5,000; cat. no. ab124964) (all from Abcam, Cambridge, UK) at $4^{\circ} \mathrm{C}$ overnight. The next day, the membrane was washed with phosphate-buffered saline with Tween-20 (PBST) 3 times (10 min per time), added with the secondary antibody (diluted at 1:2,000; cat. no. SA00001-1; ProteinTech Group, Inc., Chicago, IL, USA) for incubation at room temperature for $2 \mathrm{~h}$, and washed again with PBST 3 times (10 min per time). Finally, the ultra-sensitive luminescence solution was dropwise added, followed by photography using the gel imaging system and observation of results.

Co-immunoprecipitation. The medium in the culture dish was discarded, the culture dish was washed with $1 \mathrm{X}$ pre-cooled PBS 3 times, and added with $500 \mu$ IP lysis solution containing protease inhibitor in each dish, followed by lysis on ice for $30 \mathrm{~min}$. Cells were scraped off using the cell scraper, blown and beaten repeatedly until they shed completely followed by centrifugation at $12,000 \mathrm{xg}$ at $4^{\circ} \mathrm{C}$ for $15 \mathrm{~min}$. The supernatant was transferred into a new Eppendorf (EP) tube, and the protein concentration was determined using the BCA method. Whole protein lysis solution $(50 \mu \mathrm{m})$ was taken as the input, $500 \mu \mathrm{g} / \mathrm{mg}$ whole protein lysis solution was taken, added with $20 \mu \mathrm{l}$ Protein $\mathrm{G}$ magnetic beads, and rotated at $4^{\circ} \mathrm{C}$ for $1-2 \mathrm{~h}$. The tube was put on the magnetic shelf, and the supernatant was transferred into a new EP tube as the pre-cleaning. IgG $(2 \mu \mathrm{g})$ antibody or target antibody NL1 was added into the supernatant, and the mixture was agitated and kept overnight at $4^{\circ} \mathrm{C}$. The next day, $20 \mu \mathrm{l}$ protein $\mathrm{G}$ immunomagnetic beads

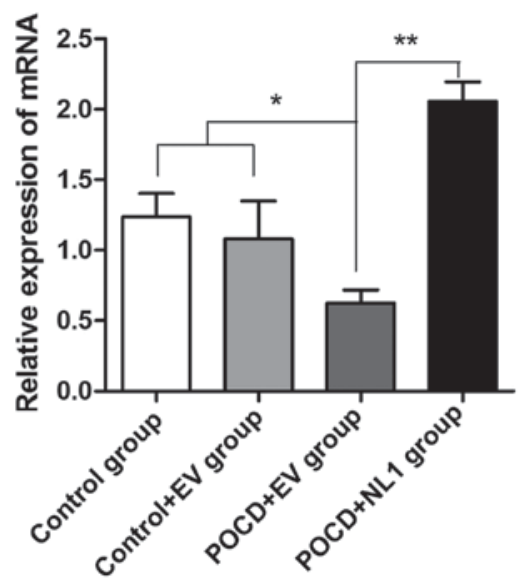

Figure 1. Detection of NL1 mRNA expression levels in control group $\mathrm{C}+\mathrm{V}$ group, $\mathrm{POCD}+\mathrm{EV}$ group and $\mathrm{POCD}+\mathrm{NL} 1$ group. ${ }^{*} \mathrm{P}<0.05,{ }^{* *} \mathrm{P}<0.01$. $\mathrm{POCD}$, postoperative cognitive dysfunction.

were added into the above mixture, and shaken at $4^{\circ} \mathrm{C}$ for $2 \mathrm{~h}$ to couple the antibody with the magnetic beads. After immunoprecipitation, the mixture was centrifuged at $3,000 \mathrm{x} g$ at $4^{\circ} \mathrm{C}$ for $3 \mathrm{~min}$, at which time the beads were centrifuged to the bottom of tube. Then the beads were washed with cell lysis solution for 3-4 times, and resuspended with $5 \mathrm{X}$ sodium dodecyl sulphate loading buffer, followed by metal bath at $100^{\circ} \mathrm{C}$ for $5 \mathrm{~min}$ to dissociate the protein in the beads, and centrifugation at $12,000 \times \mathrm{g}$ for $5 \mathrm{~min}$ at $4^{\circ} \mathrm{C}$. Finally, the supernatant was transferred into a new EP tube for western blot analysis.

Immunofluorescence. After dewaxing and rehydration with alcohol, hippocampal tissue sections of mice were permeabilized with $0.1 \%$ Triton X-100 for $10 \mathrm{~min}$, and sealed with $5 \%$ standard bovine serum albumin (BSA) for $30 \mathrm{~min}$. The postsynaptic density protein 95 (PSD95) primary antibody (diluted at 1:200; Abcam) was added dropwise onto the section at $4^{\circ} \mathrm{C}$ overnight. The next day, the sections were washed with PBST 3 times, and incubated with Alexa Fluor-labeled fluorescence secondary antibody (diluted at 1:1,000; Thermo Fisher Scientific, Inc., Waltham, MA, USA) at room temperature for $1 \mathrm{~h}$. After DAPI nuclear staining for $3 \mathrm{~min}$, sections were observed and photographed under an inverted fluorescence microscope (DM-6000B; Leica).

Statistical analysis. MedCalc software (Version 18.0.0, Mariakerke, Belgium) was used for data statistics and processing. Measurement data are presented as mean \pm standard deviation, and independent-samples t-test was used for the intergroup comparison. Chi-square test was used for enumeration data. $\mathrm{P}<0.05$ was considered to indicate a statistically significant difference.

\section{Results}

Detection of $N L 1$ messenger ribonucleic acid ( $m R N A)$ expression levels in hippocampal tissues of mice in four groups. After the experiment, hippocampal tissues were taken to detect the difference in the NL1 mRNA expression level among the three groups. As is evident from Fig. 1, levels of NL1 mRNA 


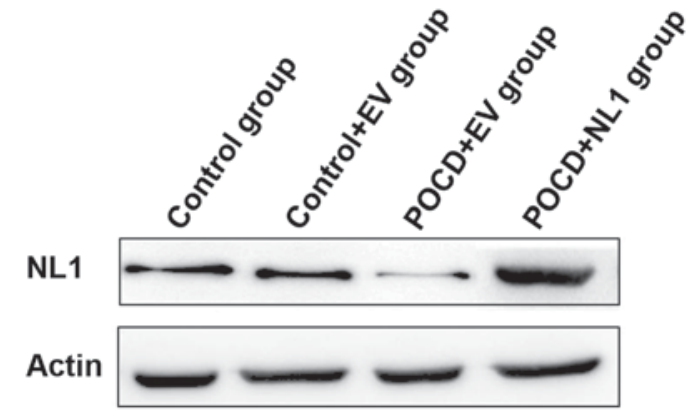

Figure 2. Detection of NL1 protein expression levels in control group , $\mathrm{C}+\mathrm{V}$ group, POCD+EV group and POCD+NL1 group. IIA: Detection of NL1 protein bands via western blotting, IIB: gray values of protein bands. POCD, postoperative cognitive dysfunction.
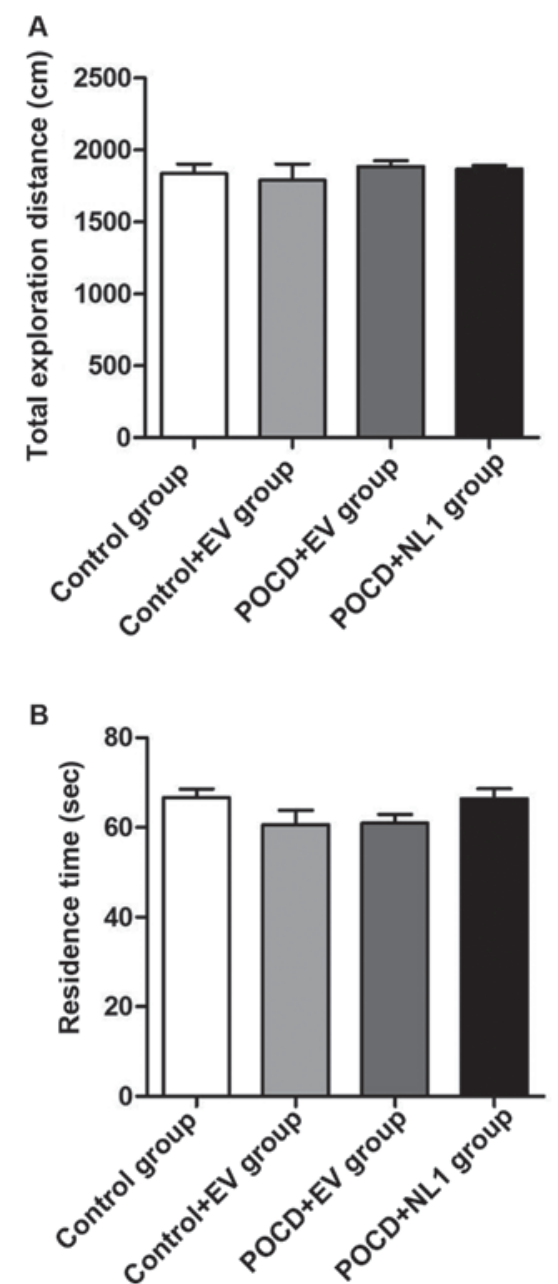

Figure 3. Comparison of total exploration distance $(\mathrm{cm})(\mathrm{A})$ and residence time in the central mesh (sec) (B) in open field test among the four groups of mice.

in the Control+EV and Control groups were not significantly different $(\mathrm{P}>0.05)$. Levels of NL1 in the POCD group were significantly lower than that in Control group $(\mathrm{P}<0.05)$, which was consistent with the downregulation of NL1 expression in hippocampal tissues of mice after POCD. Overexpression efficiency in POCD mice was detected after NL1 overexpression in vivo and compared with that in the group transfected with control plasmid in vivo. Results showed that the NL1 mRNA expression level was significantly increased $(\mathrm{P}<0.05)$ (Fig. 1).
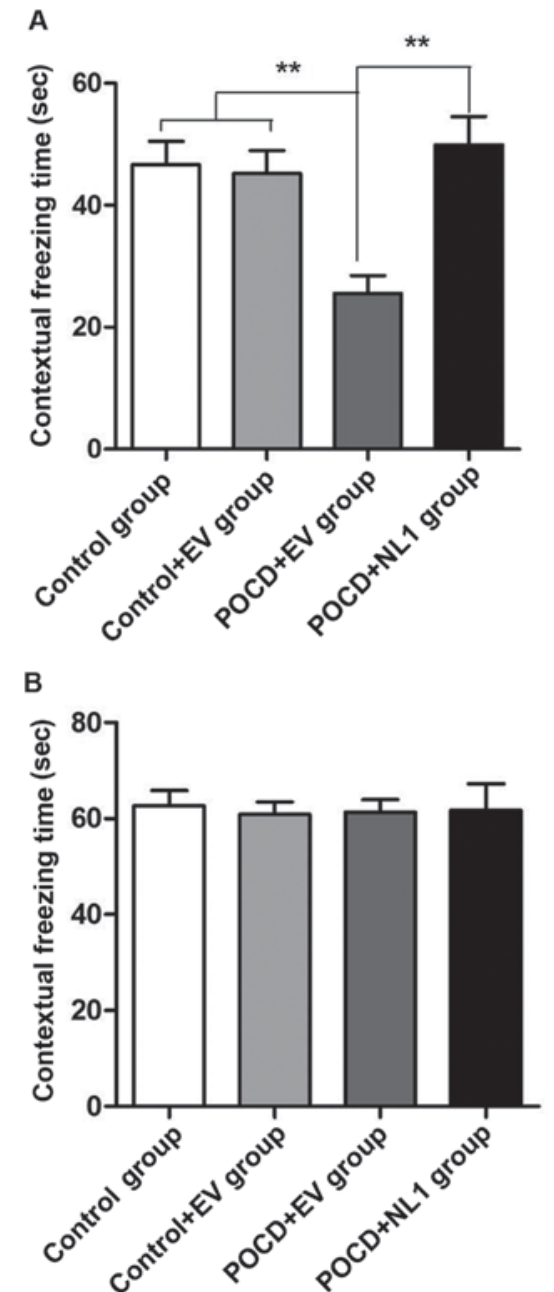

Figure 4. Comparison of freezing time in context-related fear conditioning test (A) and tone-related fear conditioning test (B) among the three groups of mice. ${ }^{* *} \mathrm{P}<0.01$

NL1 protein expression level in hippocampal tissues of mice in POCD group is decreased, while that in NL1 overexpression group is increased. The NL1 protein expression level was further detected via western blotting, and quantitative comparison was made for the gray-scale scanning of band. There was no significant difference in the NL1 protein level between the Control+EV and Control groups $(\mathrm{P}>0.05)$. It suggests that empty vector has no effect on gene expression level and can be used in subsequent experiments. Compared with Control+EV group, the expression level of NL1 protein in POCD+EV group was significantly downregulated $(\mathrm{P}<0.05)$, while the expression level of NL1 protein in POCD+NL1 group was significantly higher than that in POCD+EV group $(\mathrm{P}<0.05)$ (Fig. 2).

Detection of autonomous behavior and exploratory behavior of mice in different groups via open field test. The open field test was used to investigate the autonomous exploration behavior of animals. There were no statistically significant differences in the total exploration distance (Fig. 3A) and residence time in the central mesh (Fig. 3B) among the four groups of mice (P>0.05) (Fig. 3).

Context-related fear conditioning test and tone-related fear conditioning test. Control and Control+EV groups had no 


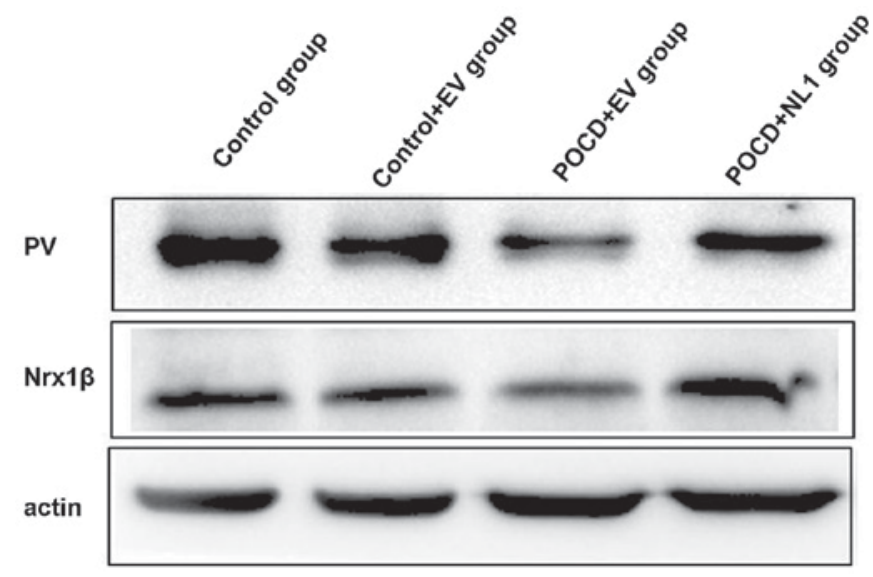

Figure 5. PV and Nrx1 $\beta$ protein expression levels in hippocampal tissues of mice in the four groups. PV, parvalbumin; $\operatorname{Nrx} 1 \beta$, neurexin- $1 \beta$.

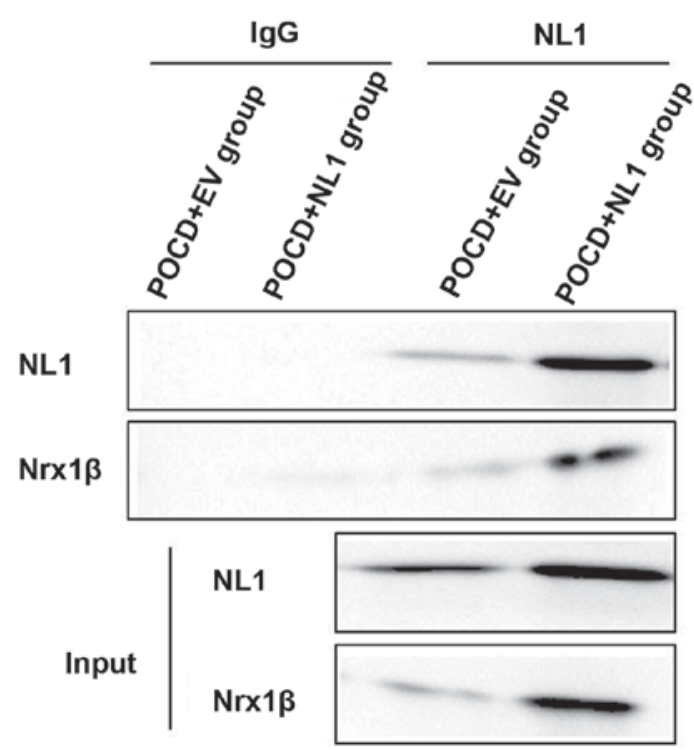

Figure 6. Interaction between NL1 and Nrx1 $\beta$ proteins in NL1 overexpression group and control group in the mouse model of POCD. POCD, postoperative cognitive dysfunction; $\operatorname{Nrx} 1 \beta$, neurexin- $1 \beta$.

significant difference in each index $(\mathrm{P}>0.05)$. Compared with Control+EV group, the percentage of contextual freezing time was obviously decreased in POCD+EV group compared with that in $\mathrm{C}+\mathrm{EV}$ group $(\mathrm{P}<0.01)$, and it was obviously increased in POCD+NL1 group compared with that in POCD+EV group $(\mathrm{P}<0.01)$, indicating that the hippocampus-dependent memory of POCD mice is greatly damaged and NL1 overexpression can repair such damage (Fig. 4A). However, there was no statistically significant difference in the cued freezing time among the three groups in the tone-related fear conditioning test $(\mathrm{P}>0.05)$ (Fig. 4B), suggesting that anesthesia operation does not affect the non-hippocampus-dependent memory.

Detection of PV and Nrxl $\beta$ protein expression levels in hippocampal tissues of mice in the four groups. The PV and Nrx1 $\beta$ protein expression levels in hippocampus were obviously decreased in POCD+EV group compared with those in $\mathrm{C}+\mathrm{EV}$ group, and they were obviously increased in POCD+NL1 group compared with those in POCD+EV (Fig. 5).

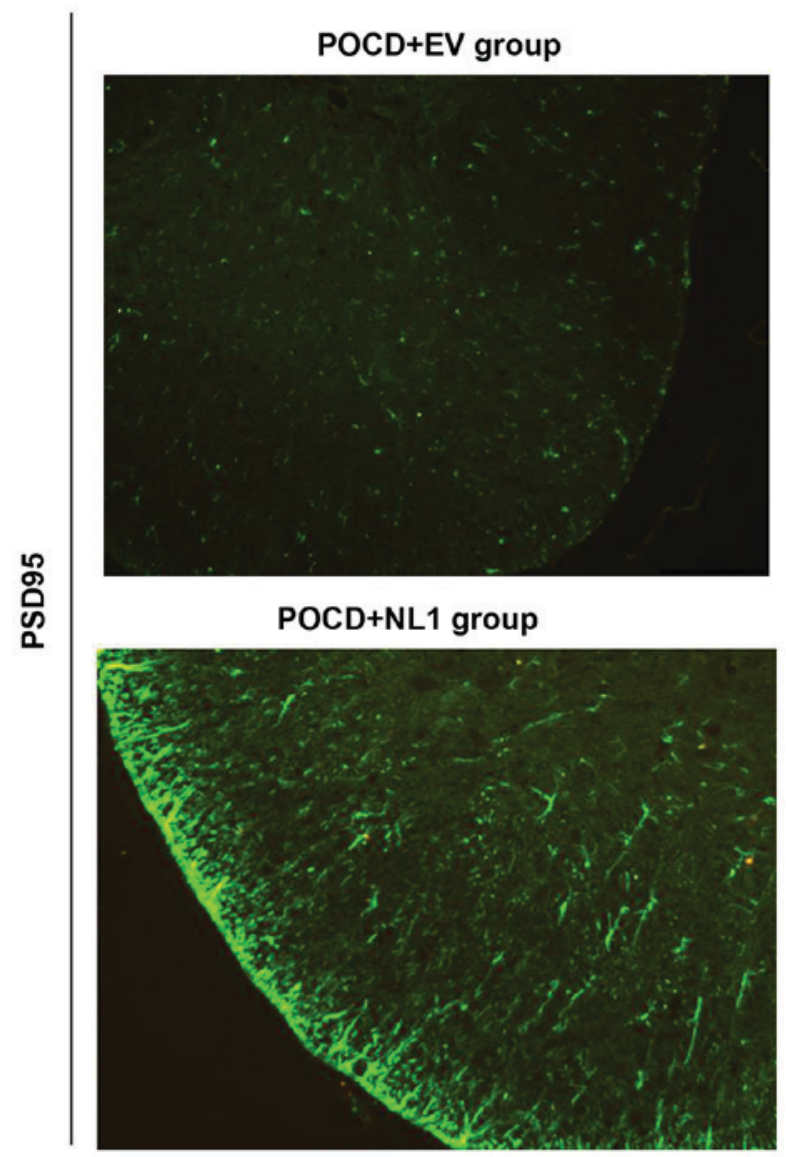

Figure 7. PSD95 expression levels in tissues in NL1 overexpression group and control group. PSD95, postsynaptic density protein 95 .

Detection of changes in interaction between NL1 and Nrxl $\beta$ via co-immunoprecipitation. NL1 is a kind of CAM specifically located on the excitatory postsynaptic membrane, which binds to the presynaptic Nrx1 $\beta$ to play an important role in the formation and function of excitatory synapses. The enhanced binding of NL1 and Nrx1 $\beta$ was detected in POCD+NL1 group compared with POCD+EV group (Fig. 6).

Effect of NL1 overexpression on the excitability of PV neurons in the mouse model of POCD. PSD95 is an anchorin purified and identified in the excitatory postsynaptic density. NL1 binds to PSD95 through the PDZ region in the cytoplasm, and the resulting complex affects the excitatory synaptic transmission via the direct interaction with N-methyl-D-aspartic acid receptor (NMDAR) and indirect interaction with $\alpha$-amino3-hydroxy-5-methyl-4-isoxazolepropionic acid receptor (AMPAR). Changes in the expression of PSD95 were detected to reflect the excitability of PV neurons after NL1 overexpression. After NL1 overexpression in POCD mice, the expression level of PSD95 in tissues was significantly increased, suggesting the increase of PV nerve excitability (Fig. 7).

\section{Discussion}

NL1 binds to PSD95 through the PDZ region in the cytoplasm, and the resulting complex affects the excitatory synaptic transmission via direct interaction with NMDAR and indirect interaction with AMPAR (15). It has been proved in the 
literature that the recruitment of hippocampal postsynaptic AMPAR and NMDAR can be decreased after NL1 knockout, thus reducing the excitatory synaptic transmission (16). NL1 affects not only the excitatory synaptic transmission through mediating the recruitment of postsynaptic glutamate receptor, but also the excitatory synaptic function through regulating the release of presynaptic glutamate (17). The knockout or decreased expression of NL1 causes damage to synaptic plasticity, and leads to memory impairment, which is involved in the occurrence of a variety of brain dysfunction-related diseases (18).

The downregulation of NL1 in the hippocampus can mediate the recruitment of postsynaptic glutamate receptor and regulate the release of presynaptic glutamate, affecting the excitatory synaptic transmission, and destroying the excitatory signals sent from pyramidal cells to PV internuncial neurons. As a result, the function of PV internuncial neurons is damaged and the inhibition of pyramidal cells declines, thus causing the imbalance of hippocampal excitatory/inhibitory synaptic transmission, and leading to $\operatorname{POCD}(19,20)$.

In this study, elderly mice were selected as subjects of study. The mouse model of POCD was constructed via isoflurane anesthesia and exploratory laparotomy, which is the commonly used mehod. NL1 was significantly upregulated in the mouse model of POCD in vivo, and the overexpression efficiency was verified at both mRNA and protein levels. There were no statistically significant differences in the movement distance and activity time in the central region in the open field test among groups, suggesting that isoflurane anesthesia does not affect the exercise and exploration abilities of mice. Fear conditioning test is a kind of sensitive method to study the learning and memory of small rodents (rats and mice) under fear conditioning. After fear conditioning training, context-related memory test and tone-related memory test were performed for experimental animals, respectively, to display the hippocampus-dependent and non-hippocampus-dependent learning and memory, respectively. Results in this study manifested that the percentage of contextual freezing time was obviously decreased in POCD+EV group compared with that in $\mathrm{C}+\mathrm{V}$ group, but there was no statistically significant difference in the cued freezing time among the three groups in the tone-related fear conditioning test, indicating that anesthesia operation does not affect the non-hippocampus-dependent memory. Therefore, it is speculated that isoflurane anesthesia can lead to the early hippocampus-dependent cognitive impairment, thus resulting in POCD.

$\mathrm{PV}$ and Nrx1 $\beta$ protein expression levels in hippocampal tissues of mice in the four groups, and the interaction between NL1 and Nrx1 $\beta$ were detected via western blotting. Results demonstrated that Control group and Control+EV group had no significant difference in each index. Compared with Control+EV group, the NL1 protein level of POCD group decreased, while the expression of NL1 protein in POCD+NL1 group was overexpressed at the same time. Interaction between NL1 and Nrx1 $\beta$ was enhanced. Furthermore, the expression level of PSD95, a postsynaptic marker of excitatory synapse, was detected. The expression level of PSD95 in POCD+NL1 group was obviously increased compared with that in POCD+EV group, indicating that the excitability on $\mathrm{PV}$ internuncial neurons is increased after NL1 overexpression in POCD mice, thus restoring the hippocampus-dependent memory and cognitive impairment in POCD.

In conclusion, this study suggests that NL1 overexpression can upregulate the expression levels of PV and Nrx $1 \beta$ in POCD mice and strengthen the interaction between NL1 and Nrx1 $\beta$, thereby further enhancing the excitability on PV internuncial neurons, which may provide new thoughts for the research on pathogenesis and prevention and treatment of POCD.

\section{Acknowledgements}

Not applicable.

\section{Funding}

No funding was received.

\section{Availability of data and materials}

The datasets used and/or analyzed during the present study are available from the corresponding author on reasonable request.

\section{Authors' contributions}

MT and YZ drafted the manuscript. MT, YZ, LQ and FL collected and interpreted the data. FL and LJ revised the manuscript. LJ, WL and LZ were responsible for the conception and design of the study. All authors read and approved the final manuscript.

\section{Ethics approval and consent to participate}

The study was approved by the Ethics Committee of Nanjing General Hospital (Nanjing, China).

\section{Patient consent for publication}

Not applicable.

\section{Competing interests}

The authors declare that they have no competing interests

\section{References}

1. Hanning CD: Postoperative cognitive dysfunction. Br J Anaesth 95: 82-87, 2005.

2. Moller JT, Cluitmans P, Rasmussen LS, Houx P, Rasmussen H, Canet J, Rabbitt P, Jolles J, Larsen K, Hanning CD, et al; International Study of Post-Operative Cognitive Dysfunction: Long-term postoperative cognitive dysfunction in the elderly ISPOCD1 study. ISPOCD investigators. Lancet 351: 857-861, 1998.

3. Rasmussen LS, Johnson T, Kuipers HM, Kristensen D, Siersma VD, Vila P, Jolles J, Papaioannou A, Abildstrom H, Silverstein JH, et al; ISPOCD2 (International Study of Postoperative Cognitive Dysfunction) Investigators: Does anaesthesia cause postoperative cognitive dysfunction? A randomised study of regional versus general anaesthesia in 438 elderly patients. Acta Anaesthesiol Scand 47: 260-266, 2003.

4. Newman S, Stygall J, Hirani S, Shaefi S and Maze M: Postoperative cognitive dysfunction after noncardiac surgery: A systematic review. Anesthesiology 106: 572-590, 2007. 
5. Krenk L, Rasmussen LS and Kehlet H: New insights into the pathophysiology of postoperative cognitive dysfunction. Acta Anaesthesiol Scand 54: 951-956, 2010.

6. Collins S: An introduction to the information processing components of the brain. Royal Signals and Radar Establishment, Malvern, UK, 1990. https://apps.dtic.mil/dtic/tr/fulltext/u2/ a222656.pdf

7. Godenschwege TA, Kristiansen LV, Uthaman SB, Hortsch M and Murphey RK: A conserved role for Drosophila neuroglian and human L1-CAM in central-synapse formation. Curr Biol 16 $12-23,2006$.

8. Kim S, Burette A, Chung HS, Kwon SK, Woo J, Lee HW, Kim K, Kim H, Weinberg RJ and Kim E: NGL family PSD-95-interacting adhesion molecules regulate excitatory synapse formation. Nat Neurosci 9: 1294-1301, 2006

9. Lepeta K, Lourenco MV, Schweitzer BC, Martino Adami PV, Banerjee P, Catuara-Solarz S, de La Fuente Revenga M, Guillem AM, Haidar M, Ijomone OM, et al: Synaptopathies: Synaptic dysfunction in neurological disorders - A review from students to students. J Neurochem 138: 785-805, 2016.

10. Bush JO and Soriano P: Ephrin-B1 regulates axon guidance by reverse signaling through a PDZ-dependent mechanism. Genes Dev 23: 1586-1599, 2009

11. Fang M, Wei JL, Tang B, Liu J, Chen L, Tang ZH, Luo J, Chen GJ and Wang XF: Neuroligin-1 knockdown suppresses seizure activity by regulating neuronal hyperexcitability. Mol Neurobiol 53: 270-284, 2016.

12. Koehnke J, Jin X, Budreck EC, Posy S, Scheiffele P, Honig B and Shapiro L: Crystal structure of the extracellular cholinesteraselike domain from neuroligin-2. Proc Natl Acad Sci USA 105 1873-1878, 2008.

13. Martínez-Guijarro FJ, Blasco-Ibáñez JM and López-García C: Postnatal increase of GABA- and PV-IR cells in the cerebral cortex of the lizard Podarcis hispanica. Brain Res 634: 168-172, 1994.
14. Dicpinigaitis PV and Dobkin JB: Antitussive effect of the GABA-agonist baclofen. Chest 111: 996-999, 1997.

15. Budreck EC, Kwon OB, Jung JH, Baudouin S, Thommen A Kim HS, Fukazawa Y, Harada H, Tabuchi K, Shigemoto R, et al: Neuroligin-1 controls synaptic abundance of NMDA-type glutamate receptors through extracellular coupling. Proc Natl Acad Sci USA 110: 725-730, 2013.

16. Chubykin AA, Atasoy D, Etherton MR, Brose N, Kavalali ET, Gibson JR and Südhof TC: Activity-dependent validation of excitatory versus inhibitory synapses by neuroligin-1 versus neuroligin-2. Neuron 54: 919-931, 2007.

17. Carpentier M,Marcinkiewicz M,Boileau G and DesGroseillers L: The neuropeptide-degrading enzyme NL1 is expressed in specific neurons of mouse brain. Peptides 24: 1083-1091, 2003.

18. Paraoanu LE, Becker-Roeck M, Christ E and Layer PG: Expression patterns of neurexin-1 and neuroligins in brain and retina of the chick embryo: Neuroligin-3 is absent in retina. Neurosci Lett 395: 114-117, 2006.

19. Savory JGA, Hsu B, Laquian IR, Giffin W, Reich T, Haché RJ and Lefebvre YA: Discrimination between NL1- and NL2-mediated nuclear localization of the glucocorticoid receptor. Mol Cell Biol 19: 1025-1037, 1999.

20. Cline H: Synaptogenesis: A balancing act between excitation and inhibition. Curr Biol 15: R203-R205, 2005.

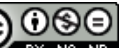

This work is licensed under a Creative Commons

Attribution-NonCommercial-NoDerivatives 4.0 International (CC BY-NC-ND 4.0) License. 